\title{
Cuisine aphrodisiaque et stratégie conjugale
}

Un mari de Syros d'Emmanuel Roïdis

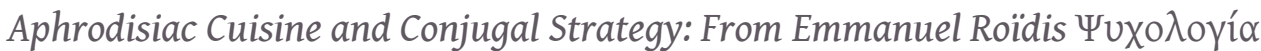



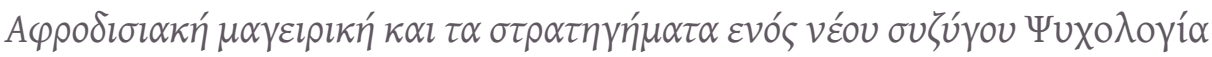

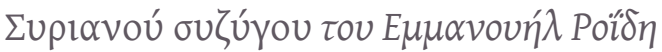

\section{Jacques Bouchard}

\section{CpenEdition}

Journals

Édition électronique

URL : https://journals.openedition.org/ceb/6496

DOI : 10.4000/ceb.6496

ISSN : 2261-4184

Éditeur

INALCO

Édition imprimée

ISBN : 978-2-85837-230-6

ISSN : 0290-7402

Référence électronique

Jacques Bouchard, «Cuisine aphrodisiaque et stratégie conjugale », Cahiers balkaniques [En ligne], Hors-série | 2016, mis en ligne le 16 mars 2017, consulté le 06 juillet 2021. URL : http:// journals.openedition.org/ceb/6496 ; DOI : https://doi.org/10.4000/ceb.6496

Ce document a été généré automatiquement le 6 juillet 2021.

Cahiers balkaniques est mis à disposition selon les termes de la Licence Creative Commons Attribution - Pas d'Utilisation Commerciale 4.0 International. 


\title{
Cuisine aphrodisiaque et stratégie conjugale
}

\author{
Un mari de Syros d'Emmanuel Roïdis

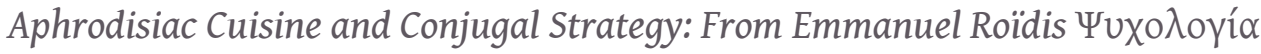

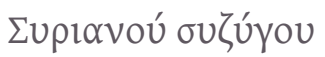

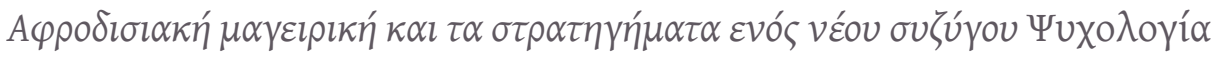

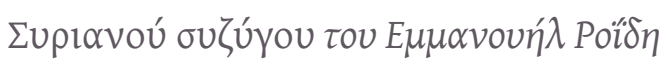

\section{Jacques Bouchard}

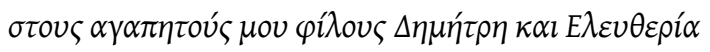

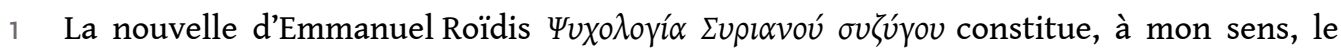
chef-d'œuvre de la littérature écrite en langue savante ${ }^{1}$. Elle a été publiée dans le

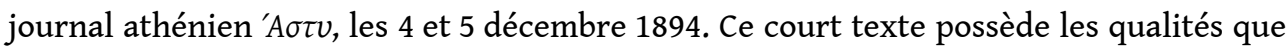
l'on trouve dans la Papesse Jeanne, sans en avoir pourtant les excès stylistiques. Florence Lozet a traduit magistralement cette nouvelle en français sous le titre d'Un mari de Syros ${ }^{2}$.

Dans ce récit, Roïdis se propose de faire l'analyse de la jalousie naissante chez un jeune époux, de même que la satire amusée de la société provinciale d'Hermoupolis, la capitale de Syros, où "tout le monde se connaît comme les moines d'un même monastère $»^{3}$.

\section{Les craintes d'un époux amoureux}

2 Le narrateur nous confesse d'entrée de jeu qu'après huit mois de mariage il est toujours amoureux de sa femme. Cette boutade est la conclusion de l'histoire qu'il va nous raconter. Le protagoniste - dont on ne connaît pas le nom - est confronté au dilemme suivant : il est follement amoureux de Christine, une gente Smyrniote d'environ vingtsix ans, au point d'en perdre le sommeil et l'appétit. La solution serait simple : épouser Christine! Mais les parents du jeune homme ne sont pas d'accord : la belle est enfant 
unique, et... sans dot. Par ailleurs, le jeune homme ne veut pas se départir des amis qu'il a l'habitude de rencontrer au club pour jouer au bridge et régler le sort du monde. Heureusement, la bonne fortune sourit au jeune Syriote: un vieil oncle part pour l'autre monde en lui laissant un petit héritage. Il décide donc d'épouser sa dulcinée et de l'emmener en voyage de noces à Kéa. Ils y passeront quatre mois à savourer les joies du mariage.

"J'épousai Christine comme on prend de la quinine, pour se débarrasser de la fièvre "4, avouera le narrateur. Une fois repu, le jeune marié croyait pouvoir enfin revenir à ses bonnes vieilles habitudes. C'était sans compter avec l'imprévu: un jour, le maire d'Hermoupolis organise un bal en l'honneur du ministre de la Marine en visite dans la région ${ }^{5}$. C'est alors que la vraie personnalité de Christine se manifeste. Elle est entièrement préoccupée par ses toilettes et son coiffeur et elle court à ces mondanités en y entraînant son mari. Or, elle y a un succès qui dépasse toute prévision: les danseurs de tout poil font la queue pour avoir le privilège de faire tourner la dive Christine. Étonné, notre narrateur l'observe, d'abord admiratif, mais rapidement déçu d'être contraint de faire tapisserie - car ce n'est jamais son tour d'étourdir son épouse.

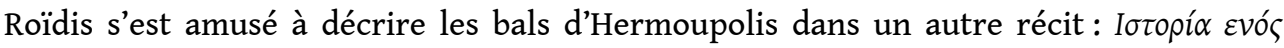
$\alpha \lambda o ́ y o v$ [Histoire d'un cheval], de 1894 aussi.

4 L'admiration fait place à la déception, puis à la suspicion, lorsqu'une fielleuse vierge quadragénaire d'insigne vertu, Cléarétie Galaxidis, lui insinue que Christine est une jeune coquette qui flirte avec tous les hommes présents. Le mari envoie paître l'insidieuse calomniatrice. Mais, renchérissant, un vieil ami au nom prédestiné, Évanghélos Haldoupis, veuf d'une épouse infidèle, conseille au pauvre mari délaissé d'accepter son sort et lui souhaite la bienvenue dans le club des cocus célèbres dont il a accroché les portraits au mur autour du sien : Agamemnon, Héphaïstos, Ménélas... Il fait remarquer à l'époux dépité qu'un jeune pharmacien, Charles Vitouris, ancien ami de Christine, récemment revenu à Syros, lui tourne autour, la mange des yeux et passe son temps à lui susurrer quelque chose à l'oreille.

5 Un texte publié en 1884, attribué peut-être abusivement à Roïdis, traite de l'infidélité de l'épouse avec beaucoup d'humour ; signée du pseudonyme le Lynx, et portant le sous-

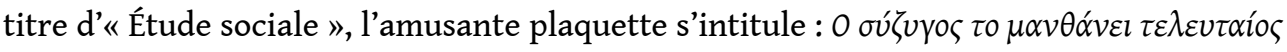
[Le mari est le dernier à l'apprendre]... ${ }^{6}$

6 Le bal tarde à finir: le couple rentre au petit matin. S'il est vrai que Christine est ravissante dans sa robe rouge cerise, elle est encore plus désirable dans sa lingerie brodée, son corset de soie... épanchant d'enivrants parfums aux fleurs d'iris et d'ylangylang. À son mari de concupiscence morfondu Christine répond : «Je suis éreintée, mon pauvre ami, je n'en puis plus... Pas ce soir, je t'en prie... $»^{7}$ La narration et les descriptions du protagoniste sont agrémentées de commentaires de l'auteur qui manifeste une connaissance subtile du cœur humain : « Les amoureux ont entre autres singularités celle d'être incapables de comprendre qu'ils sont susceptibles d'être affamés même en étant rassasiés, et qu'ils peuvent se rassasier quand ils sont affamés $»^{8}$.

7 Le mari et la femme avaient choisi de faire chambre à part, comme étant plus aristocratique. Une nuit, n'y tenant plus, le mari s'immisce dans la chambre de sa femme pendant qu'elle dort. Il la contemple, hésite à troubler son sommeil, retourne sur ses pas. En un mois, cette année-là, on ne donna pas moins de onze soirées dansantes. L'époux négligé souffre de voir sa femme se frotter aux épaules des 
danseurs, jeunes et vieux. De plus, il constate qu'elle fréquente plus que nécessaire la pharmacie du jeune Vitouris. Heureusement, il y eut un jour où le bal fut contremandé à cause d'une pluie diluvienne. Par amour certes, par désœuvrement peut-être, Christine vint offrir à son époux ce qu'il désirait tant et le déchaînement des passions fut couvert par l'orage qui grondait dehors.

Le hasard a voulu qu'à son décès le vieil oncle ait laissé en héritage à son neveu un billet gagnant de la loterie de Hambourg. Dès qu'il s'en aperçut, la première réaction de l'heureux homme fut de courir annoncer la bonne nouvelle à Christine, mais elle était sortie. Quelle chance ! Le veinard se ravisa. Roïdis commente la décision du jeune époux de n'en rien faire en ces termes: «La condition nécessaire à l'harmonie dans la vie commune avec une femme coquette consiste à lui cacher soigneusement deux choses : les neuf dixièmes de son amour, et la moitié, au moins de sa fortune $»^{9}$.

Revenu de Hambourg confortablement nanti, le mari décide donc de reprendre les choses en main. D'abord, il quitte son emploi pour se mettre à son compte, mais en fait, pour se rendre toujours disponible. Sous prétexte que le toit a des fuites, il va méthodiquement créer chez lui une ambiance et un intérieur susceptibles de retenir sa femme dans l'intimité du foyer, et, qui sait, peut-être, lui redonner le goût des fêtes charnelles. Il requiert les services d'un réfugié italien, ancien décorateur de la Scala de Milan ${ }^{10}$. Celui-ci composa un décor romantique où, comme dit le poète, "tout n'est qu'ordre et beauté, luxe, calme et volupté ». S'inspirant de l'opéra Zaira de Bellini, l'artiste transforma la chambre en une alcôve de style franco-ottoman, y ajouta de lourdes tentures, des tissus cousus de fils d'or repiqués de vieux vêtements sacerdotaux, y plaça la vasque d'un ancien baptistère byzantin, et coiffa le tout d'un luminaire qui répandait une lumière de saphir tamisée. L'époux transi édifia ainsi un temple à l'amour, dont la capiteuse splendeur serait habitée par son idole, la sémillante Christine.

Pour distraire son épouse et la garder au foyer, il lui offre des pots de camélias, un piano droit, un stéréoscope, un chat angora. Il lui fait suivre des cours de chant à domicile. Pour mieux prouver son amour, il la comble de cadeaux. Il l'abonne à la Chronique élégante et à la Vie parisienne, des revues qui lui permettent de suivre la mode de la Ville Lumière et de commander des toilettes magnifiques pour épater la petite société de l'île, surtout les femmes.

\section{La cuisinière milanaise de l'évêque}

Mais le point culminant de la stratégie conjugale élaborée par l'inventif mari de Christine est d'un tout autre ordre : son succès majeur est d'avoir réussi à suborner la cuisinière milanaise de l'évêque catholique d'Ano Syra, dont la réputation de maîtressequeux était bien connue dans les Cyclades. Elle met au profit de ses nouveaux maîtres tout son savoir-faire dans la préparation d'un menu directement issu, dirait-on, d'un bréviaire de cuisine aphrodisiaque : d'abord des conventionnels raviolis - dont l'auteur passe la recette sous silence - ; je me plais à penser qu'il s'agit vraisemblablement des raviolis d'huîtres, comme le suggère le cuisinier montréalais Giovanni Apollo, dans son manuel intitulé Recettes interdites ${ }^{11}$. J'en arrive à cette conclusion parce que le narrateur mentionne les effluves d'huîtres. De plus, notre cuisinière expérimentée sert au jeune couple une soupe de crevettes et son fameux cappon magro, inventé, paraît-il, par le pape Clément XIV (1705-1774) : il ne s'agit pas d'un chapon maigre, comme son nom 
semble l'indiquer. C'est un plat relevé d'épices, de moules, de crevettes et d'autres fruits de mer. Roïdis a pu en lire la recette dans le fameux guide culinaire de Gian Battista Ratto : la Cuciniera genovese ossia la vera maniera di cucinare alla genovese, dont la $8^{e}$ édition parut justement à Gênes en $1893^{12}$. Tous les livres de recettes chantent les vertus des épices : elles exercent une action vasodilatatrice sur les organes génitaux et aiguisent l'appétit sexuel. Quant aux poissons et aux fruits de mer, on sait qu'ils sont riches en zinc: cet oligo-élément intervient dans le processus de synthèse de la testostérone. Casanova ingurgitait une quantité impressionnante d'huîtres avant d'honorer une dame ${ }^{13}$.

12 La cuisinière milanaise connaissait fort bien le pouvoir fortifiant des œufs ; cette sainte femme savait que leur cuisson correspondait exactement au temps qu'il faut pour réciter deux Ave Maria. Elle avait des secrets de préparation tout à fait personnels, ainsi pour le rouget: dès sa sortie du filet, on devait l'achever immédiatement au moyen d'une aiguille, pour empêcher qu'une longue agonie n'altérât sa saveur. Elle cuisinait avec toutes sortes d'herbes aromatiques dans un jus d'orange amère. Elle devait utiliser, certes, le basilic, comme de tout temps en Méditerranée on en use, pour concocter des philtres d'amour. La ciboulette et l'oignon ne devaient pas manquer non plus : ils réchauffent les reins et sont indiqués, paraît-il, pour combattre la frigidité féminine ${ }^{14}$. Quand elle préparait une dinde, elle la nourrissait trois jours durant de noix de muscade avant de l'occire. La noix de muscade est un puissant aphrodisiaque qui dégage une odeur qui provoque le vertige, mais aussi, la chaîne alimentaire aidant, un afflux de sang dans les organes génitaux. Ces agapes étaient accompagnées de deux ou trois verres de vin qui, faisant disparaître les inhibitions, préparaient la voie vers la couche conjugale. Le narrateur a raison de mentionner une quantité raisonnable de boisson alcoolisée : boire davantage affaiblit la libido et conduit prématurément au sommeil, tous les connaisseurs en conviennent.

Le mari résume admirablement l'atmosphère festive qui règne chez lui, lorsqu'il loue

[...] cette félicité que fait naître la satisfaction simultanée de tous nos sens autour d'une table luxueuse, de la chaleur d'un bon feu, des reflets de la lumière sur le service d'argent et de cristal, du parfum des fleurs, de l'odeur marine des huîtres, avec deux ou trois verres d'un vieux vin et la présence d'une jeune femme dont le visage peu à peu s'anime et le regard s'allume ${ }^{15}$.

14 Certes, l'hiver ramena inévitablement les soirées dansantes à Syros, mais le mari savait dorénavant que sa femme n'avait nulle intention de le coiffer des bois de Ménélas et d'Héphaïstos : ce qu'elle désirait, c'était capter l'admiration de la gent masculine, mais surtout éblouir les dames d'Hermoupolis.

\section{Conclusion}

15 Une question pourtant demeure : indigné de s'être fait souffler aussi cavalièrement sa maitresse-queux italienne, l'évêque catholique accusa notre brave mari orthodoxe de prosélytisme. Roïdis n'en dit rien, mais se gausse en sourdine : comment le digne prélat pouvait-il se délecter d'une cuisine aussi relevée, appropriée certes pour un charmant couple de nouveaux mariés, mais susceptible de mettre en péril la santé, la sérénité, voire le salut, du vénérable monseigneur? 


\section{NOTES}

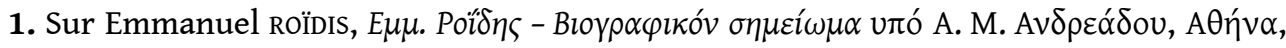
1911.

2. Emmanuel RoÏDIS, Un mari de Syros, Arles, Institut français d'Athènes - Actes Sud, traduit du grec par Florence Lozet.

3. Ibidem, p. 17.

4. Ibid., p. 10-11.

5. Dans la seconde moitié du XIX ${ }^{\mathrm{e}}$ siècle, Syros fut un port international, le premier de Grèce.

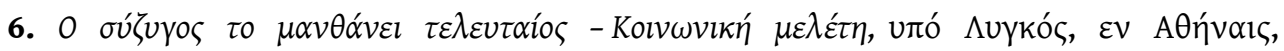

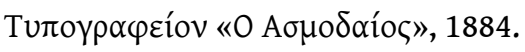

7. Emmanuel RoÏDIS, Un mari de Syros, op.cit., p. 22.

8. Ibid.

9. Emmanuel RoÏDIs, Un mari de Syros, op.cit., p. 33.

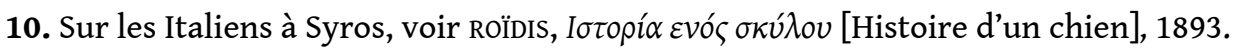

11. Montréal, Éditions Transcontinental, 2009.

12. p. 216-218. Né à Syros en 1836, Roïdis vécut à Gênes de 1841 à 1849. Je remercie chaleureusement mesdames Cristina Formenton et Maria Roussou, de l'Université de Chypre, pour leur aide et leurs suggestions.

13. Marie-Amélie PICARD, les Aliments du désir, Paris, Éd. Trajectoire, 2003, p. 182.

14. Voir Giovanni APOLLO, op.cit., p. 188.

15. Emmanuel Rö̈DIs, Un mari de Syros, op.cit., p. 37.

\section{RÉSUMÉS}

Dans une nouvelle publiée en décembre 1894, Emmanuel Roïdis expose la stratégie d'un jeune mari désireux de reconquérir son épouse trop populaire auprès des danseurs de Syros. Il la comble donc de parures et de frivolités, lui édifie un décor intimiste, mais de plus, il s'attache la cuisinière milanaise ci-devant au service de l'évêque catholique de l'île. La maîtresse queux élabore une cuisine apte à aiguiser la sensualité du couple et à raffermir les liens du mariage.

In a novella published in December 1894, the Modern Greek author Emmanuel Roïdis presents the stratagem devised by a young husband seeking to reconquer the heart of his wayward wife, who has become far too popular with the dancers of Syros. The jealous spouse lavishes upon her fineries and frivolities, ensconces her in the most intimate surroundings, and engages for her the Milanese chief cook of the island's Catholic archdiocese whose skillful hands send forth a variety 
of delicacies calculated to sharpen the couple's sensual proclivities and to enhance the marriage bonds.

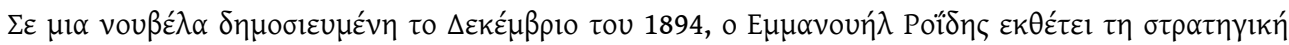

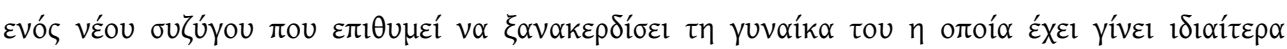

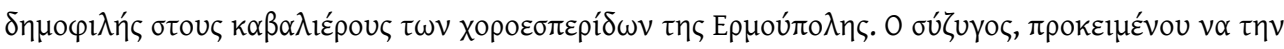

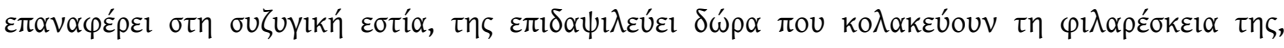

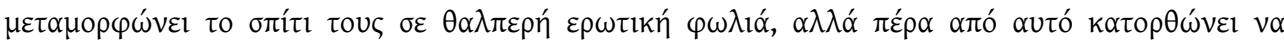

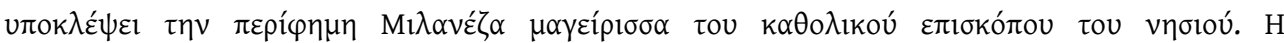

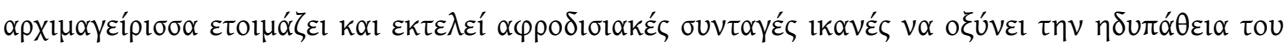

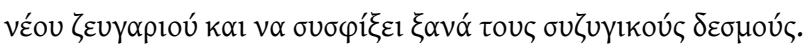

\section{INDEX}

Index géographique : Grèce

motsclestr Yemek, afrodizyak, evlilik ilişkileri, Roidis Emmanuil (1836-1904), Yunanistan, On dokuzuncu yüzyıl, Edebiyat

Mots-clés : cuisine, cuisine, aphrodisiaque, relation conjugale, relation conjugale, Roïdis Emmanuel (1836-1904), Roïdis Emmanuel (1836-1904)

motsclesmk ГАСТРОНОМИЈА, АФРОДИЗИЈАК, БРАЧНИ ОДНОСИ, РОИДИС ЕМАНУЕЛ (1836-1904), ГРЦИЈА, ДЕВЕТНАЕСЕТТИОТ ВЕК, ЛИТЕРАТУРА

Keywords : Cooking, Aphrodisiac, Conjugal relations, Roïdis Emmanuel (1836-1904), Nineteenth Century, Literature

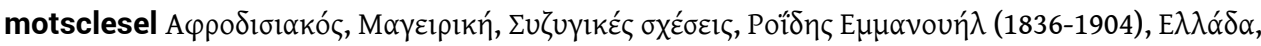

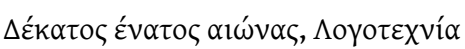

Thèmes : littérature

Index chronologique : dix-neuvième siècle

\section{AUTEUR}

\section{JACQUES BOUCHARD}

Université de Montréal 